\title{
Prognostic factors for colectomy in refractory ulcerative colitis treated with calcineurin inhibitors
}

\author{
SHIGEKI BAMBA ${ }^{1}$, AKIRA ANDOH ${ }^{2}$, HIROTSUGU IMAEDA ${ }^{1}$, HIROMITSU BAN ${ }^{1}$, AYAKO KOBORI ${ }^{1}$, \\ YOUSUKE MOCHIZUKI $^{3}$, MAKOTO SHIOYA ${ }^{1}$, TAKASHI NISHIMURA ${ }^{1}$, OSAMU INATOMI ${ }^{1}$, \\ MASAYA SASAKI ${ }^{4}$, YASUHARU SAITOH ${ }^{3}$, TOMOYUKI TSUJIKAWA ${ }^{5}$ and YOSHIHIDE FUJIYAMA ${ }^{1}$ \\ ${ }^{1}$ Division of Gastroenterology; ${ }^{2}$ Division of Mucosal Immunology, Graduate School of Medicine; \\ Divisions of ${ }^{3}$ Endoscopy, ${ }^{4}$ Clinical Nutrition, and ${ }^{5}$ Comprehensive Internal Medicine, \\ Shiga University of Medical Science, Otsu, Shiga, Japan
}

Received December 6, 2011; Accepted March 15, 2012

DOI: $10.3892 /$ etm.2012.545

\begin{abstract}
Calcineurin inhibitors (CNIs) such as cyclosporin A (CSA) and tacrolimus (FK506) are efficacious in patients with steroid-refractory or steroid-dependent ulcerative colitis (UC). We retrospectively investigated patients with refractory UC treated with CNIs to elucidate the prognostic factors for a colectomy. Data from 59 patients ( 35 men and 24 women) were analyzed. CSA and FK506 were administered by intravenous infusion and peroral administration, respectively. The efficacy of the CNIs was assessed using Seo's complex integrated disease activity index. Categorical data analyses were also conducted. The results revealed that the response rates for CSA and FK506 were similar (CSA, 66.6\%; FK506, 63.6\%). However, oral FK506 had a slower onset of action than intravenous CSA. The risk factors for CNI non-responsiveness were: i) more than $10,000 \mathrm{mg}$ of prednisolone used prior to CNI treatment; and ii) positivity for cytomegalovirus antigenemia (C7-HRP). The factors affecting the rate of colectomy were: i) CNI non-responsiveness; ii) more than $10,000 \mathrm{mg}$ of prednisolone used prior to the initiation of CNI treatment; and iii) positivity for C7-HRP. The addition of azathioprine (AZA) following CNI treatment significantly reduced the incidence of colectomy. Our results revealed the prognostic factors affecting the efficacy of CNI therapy and the need for colectomy in patients with refractory UC. Importantly, some of these factors may be obtained prior to or shortly following the start of CNI treatment. Furthermore, AZA is an important agent for averting colectomy once a patient responds to CNIs.
\end{abstract}

Correspondence to: Dr Shigeki Bamba, Division of Gastroenterology, Shiga University of Medical Science, SetaTsukinowa, Otsu, Shiga 520-2192, Japan

E-mail: sb@belle.shiga-med.ac.jp

Key words: ulcerative colitis, calcineurin inhibitor, cyclosporin A, tacrolimus, colectomy

\section{Introduction}

Ulcerative colitis (UC) is chronic colitis of unknown etiology. Traditionally, patients who have severe attacks requiring hospitalization are treated with intravenous corticosteroids. Their response rate is approximately $60 \%$. Recently, calcineurin inhibitors (CNIs) have been used as alternative treatments for steroid-refractory or steroid-dependent UC. There are two main options for CNI induction therapy: intravenous cyclosporin A (CSA) and oral tacrolimus (FK506).

CSA blocks the calcium-dependent signal transduction pathway emanating from the T-cell receptor, thereby inhibiting the action of helper T cells. CSA is also administered to patients with graft-versus-host disease following allogeneic bone marrow transplantation or to patients undergoing renal transplant. With respect to steroid-refractory or steroid-dependent UC, CSA induction therapy provides an effective medical alternative for patients previously faced with only surgical options. Uncontrolled trials $(1,2)$ and controlled trials (3) established the efficacy of short-term CSA use as a 'rescue therapy' in severe UC. Lichtiger et al reported that intravenous CSA followed by oral therapy demonstrated an initial response rate of $82 \%$ within a mean of 7 days versus $0 \%$ in the group that received steroids alone (3).

On the other hand, tacrolimus (FK506) has similar pharmacological mechanisms of action to CSA. Fellermann et al reported the effectiveness of FK506 for refractory UC patients. FK506 was initiated either intravenously at a dose of $0.01-0.02 \mathrm{mg} / \mathrm{kg} / \mathrm{day}$ or $0.1-0.2 \mathrm{mg} / \mathrm{kg} / \mathrm{day}$ orally. They concluded that peroral dosing is equivalent to intravenous administration (4). Ogata et al reported the results of a placebo-controlled, double-blind study which revealed that oral FK506 improved disease activity for $68.4 \%$ of the patients in the high trough group as compared with $10.0 \%$ in the placebo group (5).

As described above, these two CNIs are powerful agents in bringing about remission of active UC. However, even where CNIs are administered, a consequent colectomy may be required in certain cases. We previously reported the factors affecting the efficacy of CSA therapy for refractory ulcerative colitis (6). In the present study, we conducted a retrospective 
Table I. Clinical background of the study participants.

\begin{tabular}{lll}
\hline Clinical characteristic & \multicolumn{1}{c}{ CSA } & FK506 \\
\hline Total no. of patients (M/F) & $48(28 / 20)$ & $11(7 / 4)$ \\
Age, in years; mean \pm SD (range) & $33.4 \pm 12.7(17-62)$ & $34.5 \pm 12.3(16-59)$ \\
Disease duration, in years; mean \pm SD (range) & $3.9 \pm 4.0(0.05-15.7)$ & $5.2 \pm 5.0(0.15-16.4)$ \\
Seo's CIDAI; mean \pm SD (range) & $221.1 \pm 24.7(166.0-275.5)$ & $213.2 \pm 34.8(143.0-265.7)$ \\
Undermining ulcer (present/absent) & $52.0 \%(25 / 23)$ & $36.3 \%(4 / 7)$ \\
Disease extent (pancolitis/left-sided) & $66.6 \%(32 / 16)$ & $63.6 \%(7 / 4)$ \\
C7-HRP (positive/negative) & $31.2 \%(15 / 33)$ & $18.0 \%(2 / 9)$ \\
Total PSL used before CNI treatment (mg) $;$; mean \pm SD (range) & $5,213 \pm 7,172(140-36,500)$ & $4,517 \pm 4,816(0-11,330)$ \\
CNI response rate (responders/all) & $66.6 \%(32 / 48)$ & $63.6 \%(7 / 11)$ \\
Colectomy rate (number) & $52 \%(25 / 48)$ & $36.3 \%(4 / 11)$
\end{tabular}

${ }^{a}$ All corticosteroids used were converted to PSL. CIDAI, complex integrated disease activity index; CNI, calcineurin inhibitor; CSA, cyclosporin A; PSL, prednisolone; SD, standard deviation.

study to investigate the prognostic factors for colectomy in the era of CNI treatment.

\section{Patients and methods}

Patients. We reviewed the medical charts and recent follow-up of 60 consecutive UC patients ( 35 men and 25 women) who had been initially treated with CNIs for disease flare-ups between December 1999 and March 2011 at the Shiga University of Medical Science Hospital. One case with concomitant psychological disorders was excluded due to a suspension of the CNI treatment (Table I). In general, the CNIs were administered to patients resistant to systemic corticosteroids. The cytomegalovirus infections were validated from blood samples by cytomegalovirus antigenemia (C7-HRP). Twelve out of 17 patients also received concurrent ganciclovir treatment due to cytomegalovirus infection. The average patient age was 33.6 years [standard deviation (SD) 12.5, range 16-62], and the disease type included one attack in only 8 cases, chronic continuous attacks in 14 cases and relapse remitting attacks in 37 cases. The disease extent was pancolitis in 39 cases and left-sided colitis in 20 cases. To prevent Pneumocystis carinii infection, sulfamethoxazole/trimethoprim was administered to all cases treated with the CNIs.

CSA administration. CSA was administered by continuous infusion with starting doses of $2-4 \mathrm{mg} / \mathrm{kg} /$ day for a maximum of 14 days. The serum CSA levels were monitored three times per week during the infusion therapy, and the infusion dose was altered by aiming for $350-450 \mathrm{ng} / \mathrm{ml}$. Following successful continuous CSA infusion, we switched to peroral dosing. The total peroral daily doses were double those of the continuous daily infusions. The trough serum levels were monitored, and the dose of CSA was adjusted to trough serum levels of $100-200 \mathrm{ng} / \mathrm{ml}$. The average duration of the peroral CSA administration was 145 days.

FK506 administration. FK506 treatment was started at a dose of $0.1 \mathrm{mg} / \mathrm{kg} / \mathrm{day}$, divided in two portions since a total oral daily dose of approximately $0.1 \mathrm{mg} / \mathrm{kg} /$ day was necessary

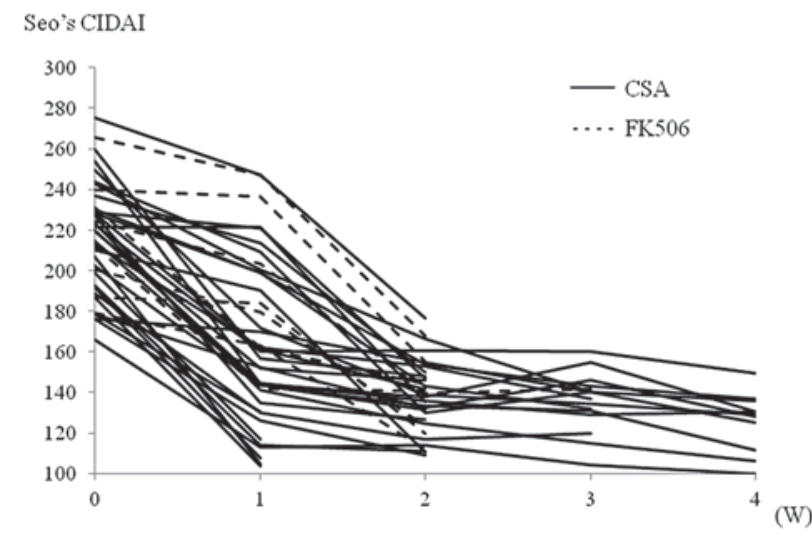

Figure 1. Clinical course following initiation of calcineurin inhibitors (CNIs). The Seo's complex integrated disease activity indices (CIDAIs) were recorded weekly during hospitalization and post-CNI administration.

to achieve a high trough level of $10-15 \mathrm{ng} / \mathrm{ml}$. The serum FK506 levels were monitored three times per week during admission. The dose was altered by aiming for a high trough level of $10-15 \mathrm{ng} / \mathrm{ml}$ for the first week, and then for a low trough level of 5-10 ng/ml for the following week. The absorption of FK506 is known to decrease by $38 \%$ following meals (7). To minimize the effect of meals, FK506 was prescribed to be taken one hour before food. The average duration of FK506 administration was 127 days.

Definitions of outcome. The disease activity was assessed by using Seo's complex integrated disease activity index (CIDAI) (8). Scores below 150 indicated cases in 'remission'. 'CNI responders' were defined as those with a 50-point decrease two weeks following CNI administration. Following the successful induction of remission, those patients who required hospitalization or additional induction therapy such as steroids, cytophoresis, infliximab and CNIs were defined as in 'relapse'.

Statistical analyses. The patient characteristics of the responders were then compared to the non-responders to 


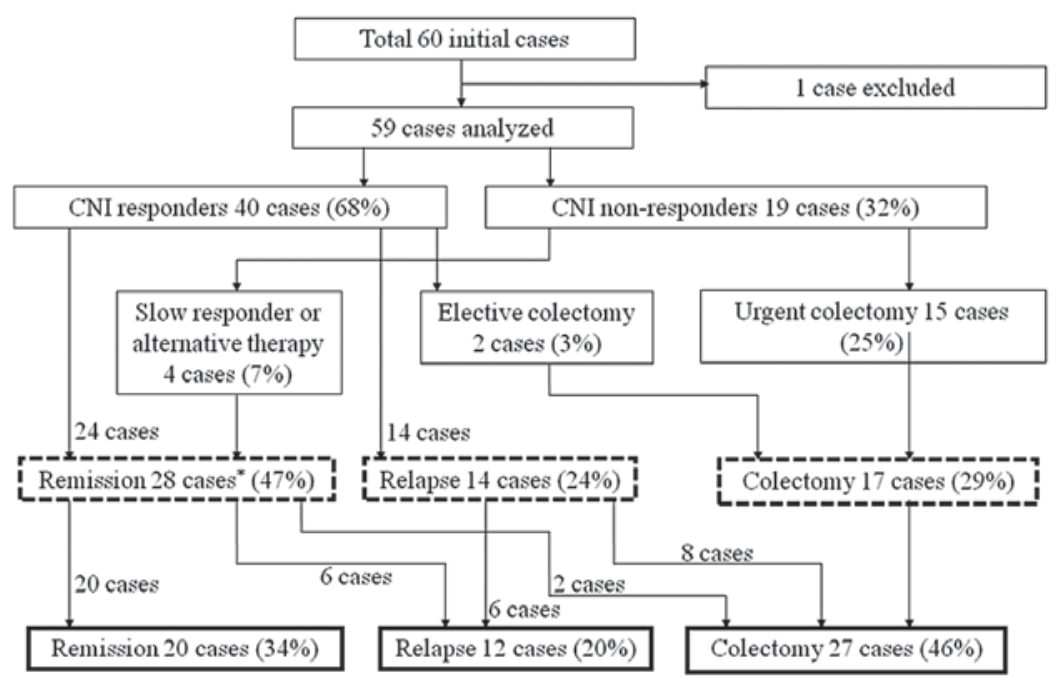

Figure 2. Flow chart illustrating the outcome of all UC patients treated with calcineurin inhibitors (CNIs). The outcomes at one year after CNI administration are shown in dashed squares. The overall outcomes are shown in bold squares. The average overall observation period was 4.6 years (SD 3.5). "Including 5 cases with a follow-up period of $<1$ year.

elucidate the characteristics of the responders. For evaluating the prognostic factors of efficacy, categorical data analyses were performed on gender, age ( $\geq 40$ years, $<40$ years), disease duration ( $\geq 4$ years, $<4$ years), Seo's CIDAI upon initiation of CSA treatment ( $\geq 220$ points, $<220$ points), endoscopic findings of undermining ulcers, disease extent (total colitis type or left-sided colitis), C7-HRP, and total prednisolone (PSL) used prior to CNI treatment ( $\geq 10,000 \mathrm{mg},<10,000 \mathrm{mg}$ ). The overall colectomy-free survival was calculated using the Kaplan-Meier method. The log-rank test was used to determine the statistical difference between groups. Receiver operating characteristic (ROC) curves were plotted to compare the prognostic yield of these factors. All statistical analyses were performed with StatView v5.0 (SAS Institute, Cary, NC, USA), GraphPad Prism v4.03 and SPSS v16.0.1 (SPSS, Chicago, IL, USA) software.

\section{Results}

Comparison between CNIs. The baseline characteristics of the study population are described in Table I. The patients' backgrounds, the effectiveness of the CNIs and the colectomy rate did not differ significantly between these two CNI groups. The only difference was the onset of action following initiation of CNI treatment. As shown in Fig. 1, the FK506 responders had a slower onset as compared to the CSA responders. The prevalence of CNI responders within one week following CNI administration was 56\% (18/32) in the CSA responder group as compared with $28 \%(2 / 7)$ in the FK506 responder group (Fig. 1). Seo's CIDAI decreased 53.1 points (SD 31.8) in the CSA responder group as compared with 28.8 points (SD 28.8) in the FK506 responder group $(\mathrm{P}=0.035)$. To achieve a high trough level of FK506, an average of 8.5 days was required.

The switching of CNI administration during single induction therapy was not effective in two cases; one from CSA to FK506, the other from FK506 to CSA. These two cases required urgent colectomies.
Table II. Risk estimates of CNI non-responsiveness.

\begin{tabular}{lccc}
\hline & & \multicolumn{2}{c}{$95 \%$ CI } \\
\cline { 3 - 4 } Characteristic & Risk estimate & Lower & Upper \\
\hline Male gender & 1.267 & 0.412 & 3.896 \\
Age $\geq 40$ years & 1.538 & 0.481 & 4.916 \\
Disease duration $\geq 4$ years & 2.308 & 0.755 & 7.055 \\
Seo's CIDAI $\geq 220$ points & 1.061 & 0.336 & 3.070 \\
Prior AZA treatment & 1.385 & 0.416 & 4.612 \\
Undermining ulcer present & 0.900 & 0.302 & 2.685 \\
Pancolitis & 2.500 & 0.701 & 8.915 \\
C7-HRP positivity & $\mathbf{1 2 . 0 0 0}$ & $\mathbf{3 . 2 0 0}$ & $\mathbf{4 4 . 9 8 8}$ \\
PSL $\geq 10,000$ mg & $\mathbf{1 2 . 3 7 5}$ & $\mathbf{3 . 1 2 2}$ & $\mathbf{4 9 . 0 4 8}$ \\
\end{tabular}

Values in bold print are statistically significant. AZA, azathioprine; CI, confidence interval; CIDAI, complex integrated disease activity index; PSL, prednisolone.

Factors affecting CNI treatment and colectomy. An overview of the outcome of patients treated with the CNIs is shown in Fig. 2. The response rate to the CNIs was $68 \%$. Forty-seven percent of all cases analyzed stayed in remission at one year following CNI administration. The Kaplan-Meier survival analysis revealed a $53.5 \%$ relapse-free survival rate among the CNI responders at one year following the CNI administration (Fig. 3A). On the other hand, $46 \%$ of all cases analyzed underwent a subsequent colectomy during the observation period. The Kaplan-Meier analysis revealed that the overall colectomy-free survival rate among the CNI responders was $50.3 \%$ during an average observation period of 3.7 years (Fig. 3B). A stratified analysis revealed that the addition of azathioprine (AZA) following the CNI treatment significantly reduced the colectomy rate $(73.3 \%$ vs. $29.7 \%$, $\mathrm{P}=0.0169)$ (Fig. 3C). 
A

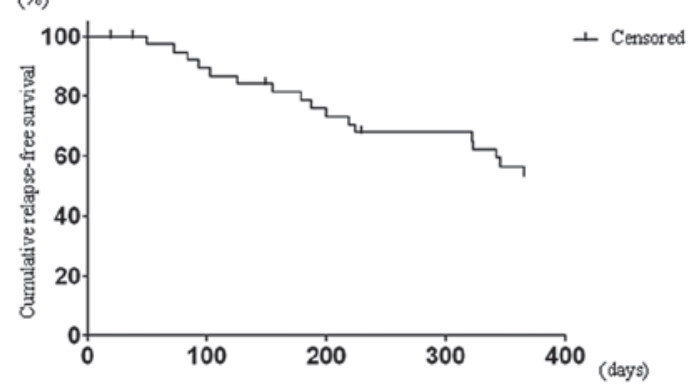

B

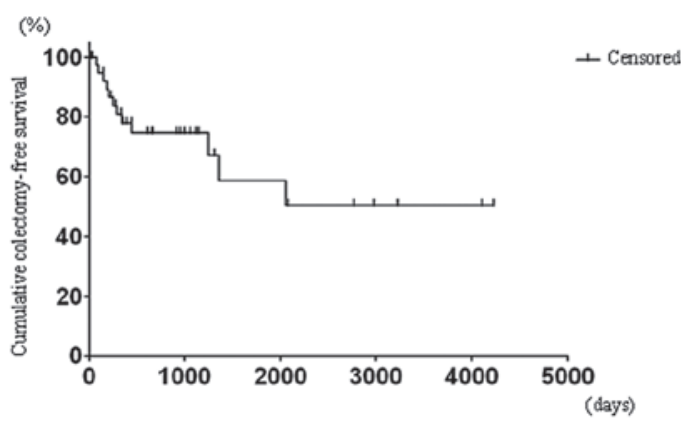

C (\%)

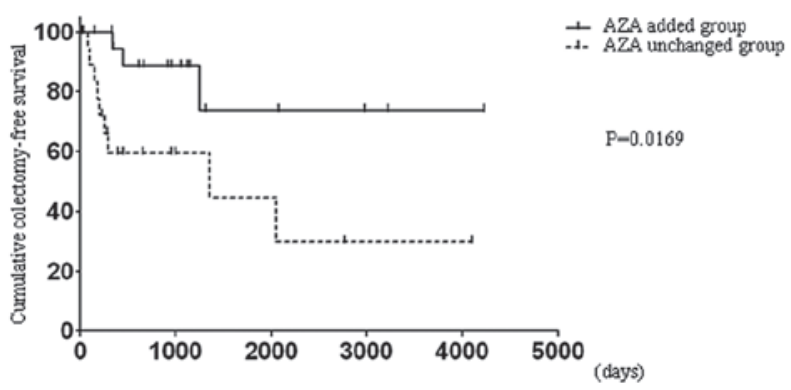

Figure 3. (A) Cumulative relapse-free survival at 1 year after CNI administration. All responders were enrolled in this analysis $(n=40)$. (B) Cumulative colectomy-free survival during the entire observation period. All responders were enrolled in this analysis $(n=40)$. (C) Stratified Kaplan-Meier analysis of the cumulative colectomy-free survival. Azathioprine (AZA) added group, $\mathrm{n}=20$; AZA unchanged group, $\mathrm{n}=20$.

As shown in Table II, the factors that lessen the efficacy of CNIs were: i) positivity for C7-HRP, and ii) more than $10,000 \mathrm{mg}$ of PSL being used prior to starting CNI treatment. Furthermore, those factors predictive of a colectomy at one year following CNI treatment were: i) CNI unresponsiveness, ii) positivity for C7-HRP and iii) more than $10,000 \mathrm{mg}$ of PSL being used prior to starting the CNIs (Table III). The same results were obtained subsequent to analyzing the prognostic factors for a consequent colectomy (Table IV).

To evaluate the prognostic yield, ROCs were plotted for a colectomy within 1 year following CNI administration (Fig. 4). The area under the curve (AUC), sensitivity and specificity were as follows: i) positivity for C7-HRP (AUC, 63.7\%; sensitivity, 47.4\%; specificity, 80\%); ii) PSL $\geq 10,000 \mathrm{mg}$ (AUC, 62.3\%; sensitivity, $42.1 \%$, specificity, $82.5 \%$ ); iii) positivity for C7-HRP + PSL $\geq 10,000$ mg (AUC, 71.4\%; sensitivity, 73.7\%; specificity, 92.5\%); and iv) CNI non-responsiveness (AUC, 84.5\%; sensitivity, $78.9 \%$; specificity, $90 \%$ ). As compared to positivity for C7-HRP alone or PSL $\geq 10,000 \mathrm{mg}$ alone, the combination of these two factors improved the prognostic yield (AUC 71.4\%).
Table III. Risk estimates of a colectomy at one year after CNI administration.

\begin{tabular}{|c|c|c|c|}
\hline \multirow[b]{2}{*}{ Characteristic } & \multirow[b]{2}{*}{ Risk estimate } & \multicolumn{2}{|c|}{$95 \% \mathrm{CI}$} \\
\hline & & Lower & Upper \\
\hline Male gender & 1.773 & 0.561 & 5.602 \\
\hline Age $\geq 40$ years & 1.077 & 0.331 & 3.506 \\
\hline Disease duration $\geq 4$ years & 0.875 & 0.284 & 2.699 \\
\hline Seo's CIDAI $\geq 220$ points & 2.800 & 0.848 & 9.245 \\
\hline Prior AZA treatment & 1.385 & 0.416 & 4.612 \\
\hline Undermining ulcer present & 0.477 & 0.156 & 1.464 \\
\hline Pancolitis & 2.500 & 0.701 & 8.915 \\
\hline C7-HRP positivity & 3.600 & 1.097 & 11.809 \\
\hline PSL $\geq 10,000 \mathrm{mg}$ & 3.429 & 1.010 & 11.643 \\
\hline CNI non-responder & 33.750 & 7.447 & 152.951 \\
\hline
\end{tabular}

Values in bold print are statistically significant. AZA, azathioprine; CIDAI, complex integrated disease activity index; CI, confidence interval: CNI, calcineurin inhibitor; PSL, prednisolone.

Table IV. Risk estimates of a consequent colectomy.

\begin{tabular}{lrrr}
\hline & & \multicolumn{2}{c}{$95 \%$ CI } \\
\cline { 3 - 4 } Characteristic & Risk estimate & Lower & Upper \\
\hline Male gender & 0.944 & 0.334 & 2.670 \\
Age $\geq 40$ years & 0.762 & 0.250 & 2.319 \\
Disease duration $\geq 4$ years & 0.514 & 0.177 & 1.491 \\
Seo's CIDAI $\geq 220$ points & 1.083 & 0.386 & 3.044 \\
Prior AZA treatment & 2.105 & 0.648 & 6.835 \\
Undermining ulcer present & 0.711 & 0.255 & 1.981 \\
Pancolitis & 2.403 & 0.788 & 7.335 \\
C7-HRP positivity & $\mathbf{3 . 5 2 9}$ & $\mathbf{1 . 0 5 1}$ & $\mathbf{1 1 . 8 5 5}$ \\
PSL $\geq 10,000$ mg & $\mathbf{3 . 9 7 2}$ & $\mathbf{1 . 0 9 1}$ & $\mathbf{1 4 . 4 6 7}$ \\
CNI non-responder & $\mathbf{1 9 . 8 3 3}$ & $\mathbf{3 . 9 5 0}$ & $\mathbf{9 9 . 5 9 0}$ \\
\hline
\end{tabular}

Values in bold print are statistically significant. AZA, azathioprine; CIDAI, complex integrated disease activity index; CI, confidence interval; CNI, calcineurin inhibitor; PSL, prednisolone.

CNI non-responsiveness was the strongest factor for predicting a colectomy within 1 year following CNI administration.

Factorial analyses for cytomegalovirus antigenemia and cumulative steroid dose were also conducted. Positivity for C7-HRP was strongly related to older age and endoscopic findings (Table V). On the other hand, cumulative steroid dose was affected by disease duration and severity (Table VI).

Ganciclovir for cytomegalovirus antigenemia and multiple administrations of CNIs. Of the 17 patients, 12 were administered ganciclovir due to cytomegalovirus antigenemia. However, ganciclovir treatment did not improve CNI responsiveness. The response rate for CNIs were $25 \%$ (3/9) in the 

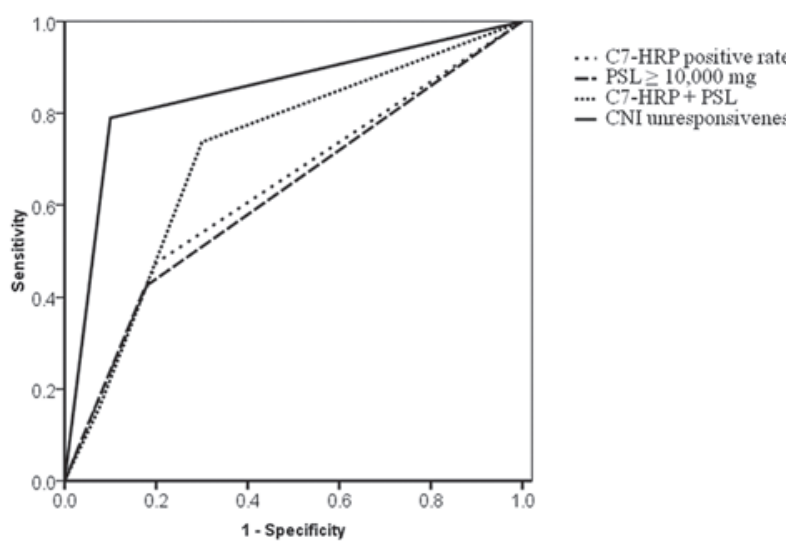
-... PSL $\mathrm{C}$-HRP+ $10,000 \mathrm{mg}$ - CNI unresponsiveness

Figure 4. Receiver operating characteristic (ROC) curves for a colectomy within 1 year after calcineurin inhibitor (CNI) administration.

Table V. Risk estimates of positivity for C7-HRP.

\begin{tabular}{lcrr}
\hline & & \multicolumn{2}{c}{$95 \%$ CI } \\
\cline { 3 - 4 } Characteristic & Risk estimate & Lower & Upper \\
\hline Male gender & 0.436 & 0.120 & 1.584 \\
Age $\geq 40$ years & $\mathbf{4 . 1 2 5}$ & $\mathbf{1 . 2 3 7}$ & $\mathbf{1 3 . 7 5 4}$ \\
Disease duration $\geq 4$ years & 0.802 & 0.249 & 2.585 \\
Seo's CIDAI $\geq 220$ points & 1.514 & 0.472 & 4.858 \\
Prior AZA treatment & 0.769 & 0.209 & 2.838 \\
Undermining ulcer present & $\mathbf{3 . 5 2 9}$ & $\mathbf{1 . 0 5 1}$ & $\mathbf{1 1 . 8 5 5}$ \\
Pancolitis & 1.429 & 0.425 & 4.801 \\
PSL $\geq 10,000 \mathrm{mg}$ & 2.000 & 0.580 & 6.898 \\
\hline
\end{tabular}

Values in bold print are statistically significant. AZA, azathioprine; CI, confidence interval; CIDAI, complex integrated disease activity index; PSL, prednisolone.

ganciclovir-treated group and 40\% (2/5) in the ganciclovirnon-treated group.

Although most patients responded to CNI induction therapy with a single course, 9 patients required multiple CNI administrations due to relapse. Seven out of these 9 patients underwent induction therapy by using CSA twice. However, none of them became quiescent following the second CSA administration, resulting in colectomies. On the other hand, 2 of these 9 patients had induction therapy by first receiving CSA and secondarily FK506. These two patients stayed in remission by taking oral FK506 as a maintenance therapy. Infliximab was used in 4 patients to treat relapses, resulting in three patients going into remission and one patient undergoing a colectomy.

Adverse events. No serious bacterial infections occurred during CNI treatment. The administration of CSA was discontinued in four cases: two cases due to liver dysfunction, and one due to renal dysfunction. An antihypertensive drug was started in the last case during CSA treatment. A magnesium agent was administered in four cases due to hypomagnesemia. FK506 was discontinued in one case due to renal dysfunction.
Table VI. Risk estimates of PSL $\geq 10,000 \mathrm{mg}$.

\begin{tabular}{lcrr}
\hline & & \multicolumn{2}{c}{$95 \%$ CI } \\
\cline { 3 - 4 } Characteristic & Risk estimate & Lower & Upper \\
\hline Male gender & 2.292 & 0.631 & 8.317 \\
Age $\geq 40$ years & 0.269 & 0.054 & 1.348 \\
Disease duration $\geq 4$ years & $\mathbf{7 . 3 3 3}$ & $\mathbf{1 . 9 5 4}$ & $\mathbf{2 7 . 5 2 8}$ \\
Seo's CIDAI $\geq 220$ points & $\mathbf{0 . 1 7 0}$ & $\mathbf{0 . 0 4 6}$ & $\mathbf{0 . 6 2 8}$ \\
Prior AZA treatment & 3.403 & 0.974 & 11.891 \\
Undermining ulcer present & 0.417 & 0.122 & 1.421 \\
Pancolitis & 0.700 & 0.208 & 2.353 \\
C7-HRP positive & 2.000 & 0.580 & 6.898 \\
& & &
\end{tabular}

Values in bold print are statistically significant. AZA, azathioprine; CI, confidence interval; CIDAI, complex integrated disease activity index.

\section{Discussion}

Prospective and retrospective studies have already reported the prognostic factors for colectomy, such as clinical, laboratory, radiological, endoscopic and genetic findings (9). In this study, the disease extent (10-13), disease duration $(14,15)$, gender $(16,17)$ and endoscopic findings $(14,15,18,19)$ were not significant predictive factors for a colectomy as well as CNI non-responsiveness, although previous reports have identified these factors to predict the outcome. Most of these previous reports investigated the prognostic factors of patients treated with corticosteroids. On the other hand, our study focused on the predictive outcomes of CNIs used to treat steroid-refractory or steroid-dependent UC. Our study population and the therapeutic agents used may have affected the results of our investigation. Almost all of our study participants were steroidrefractory or steroid-dependent. Furthermore CNIs were reported to be effective in patients with steroid-refractory or steroid-dependent UC. As the strength of treatment increases, predictive factors previously reported are thought to become insignificant.

It is natural that CNI non-responsiveness is the best predictor for a colectomy within one year following start of CNI treatment. It is particularly notable that the ROC demonstrated a moderate accuracy for colectomy prediction within one year by combining positivity for C7-HRP plus PSL $\geq 10,000 \mathrm{mg}$, since these two factors may be obtained prior to or shortly following start of the CNI treatment.

Cytomegalovirus (CMV) reactivation is known as an important exacerbating factor (20-22). However, the clinical significance of $\mathrm{CMV}$ reactivation remains controversial. Matsuoka et al claimed that the clinical significance of CMV reactivation was limited, showing that cytomegalovirus was frequently reactivated in active UC patients; however, it disappeared without antiviral agents. Therefore, they concluded that if cytomegalovirus antigenemia is low, antiviral therapies may not be necessary for most UC patients (23). On the other hand, Yoshino et al claimed that antiviral therapy was essential if $\mathrm{CMV}$ is monitored by mucosal quantitative real-time poly- 
merase chain reaction (PCR), showing that 10 (83.3\%) of the 12 patients with CMV-DNA positivity in the inflamed mucosa went into remission after applying antiviral therapy and modulating immunosuppressive therapies, and that 12 (92.3\%) of the 13 CMV-DNA-negative UC patients went into remission following treatment with more intense immunosuppressive therapies (24). Our results revealed that ganciclovir treatment did not improve the CNI responsiveness, and that C7-HRP may be used as a predictive marker for CNI non-responsiveness and for a colectomy. In this context, C7-HRP does not provide sufficient information for making a decision whether or ganciclovir should be started; however, positivity for C7-HRP indicates poor prognosis. In accordance with a previous report (25), our results also demonstrated that the patients of older age have higher risk of cytomegalovirus reactivation.

Corticosteroids are the standard therapeutic drug for UC. Our results revealed the strong connection between the longer disease duration and cumulative steroid dose. Of course, it should be taken into account that an enormous cumulative corticosteroid dose is indirectly selecting a refractory patient population. However, large amounts of steroid use may exacerbate the refractory properties of UC. Yoshiyama et al reported that cumulative steroid-overdosed UC patients may have a functional deficit in neutrophils (26). Although our study cannot determine whether the results reflect the refractory characteristics of the patients themselves or support a dysfunction of neutrophil-related immunity in steroid-overdosed patients, a prior overdose of corticosteroids was also a significant marker for CNI non-responsiveness and a colectomy.

The response rate to CSA and FK506 was similar in our results. However, FK506 had a slower onset of action than intravenous CSA. This is due to the fact that peroral FK506 required an average of 8.5 days to reach a high trough level $(10-15 \mathrm{ng} / \mathrm{ml})$ in our patients. Those patients who need urgent induction should be treated with intravenous CSA rather than oral FK506. On the other hand, once successful induction was obtained, oral FK506 may be better for maintenance therapy than oral CSA from the viewpoint of keeping stable drug serum levels. AZA is a crucial agent that maintains disease quiescence. Our results highlighted the importance of a bridging therapy to avoid a colectomy.

In conclusion, the prognostic factors for CNI efficacy and a colectomy were as follows: i) more than 10,000 mg PSL used prior to starting the CNI treatment; and ii) positivity for C7-HRP. Although the efficacy of CSA and FK506 was similar, the onset of action in induction therapy was earlier for CSA than FK506. Furthermore, the addition of AZA following successful induction therapy is important in order to avoid a colectomy.

\section{References}

1. Sandborn W: A critical review of cyclosporin therapy in inflammatory bowel disease. Inflamm Bowel Dis 1: 48-63, 1995.

2. Actis GC, Ottobrelli A, Pera A, et al: Continuously infused cyclosporine at low dose is sufficient to avoid emergency colectomy in acute attacks of ulcerative colitis without the need for high-dose steroids. J Clin Gastroenterol 17: 10-13, 1993.

3. Lichtiger S, Present DH, Kornbluth A, et al: Cyclosporine in severe ulcerative colitis refractory to steroid therapy. N Engl J Med 330: 1841-1845, 1994.
4. Fellermann K, Tanko Z, Herrlinger KR, et al: Response of refractory colitis to intravenous or oral tacrolimus (FK506). Inflamm Bowel Dis 8: 317-324, 2002.

5. Ogata H, Matsui T, Nakamura M, et al: A randomised dose finding study of oral tacrolimus (FK506) therapy in refractory ulcerative colitis. Gut 55: 1255-1262, 2006.

6. Bamba S, Tsujikawa T, Inatomi O, et al: Factors affecting the efficacy of cyclosporin A therapy for refractory ulcerative colitis. J Gastroenterol Hepatol 25: 494-498, 2010.

7. http://www.info.pmda.go.jp/downfiles/ph/PDF/800126_3999014 M1022_3_18.pdf.

8. Seo M, Okada M, Yao T, Ueki M, Arima S and Okumura M: An index of disease activity in patients with ulcerative colitis. Am J Gastroenterol 87: 971-976, 1992.

9. Travis S, Satsangi J and Lemann M: Predicting the need for colectomy in severe ulcerative colitis: a critical appraisal of clinical parameters and currently available biomarkers. Gut 60: 3-9, 2011 .

10. Meyers S, Lerer PK, Feuer EJ, Johnson JW and Janowitz HD: Predicting the outcome of corticoid therapy for acute ulcerative colitis. Results of a prospective, randomized, double-blind clinical trial. J Clin Gastroenterol 9: 50-54, 1987.

11. Truelove SC and Jewell DP: Intensive intravenous regimen for severe attacks of ulcerative colitis. Lancet 1: 1067-1070, 1974.

12. Oshitani N, Kitano A, Fukushima R, et al: Predictive factors for the response of ulcerative colitis patients during the acute-phase treatment. Digestion 46: 107-113, 1990.

13. Chakravarty BJ: Predictors and the rate of medical treatment failure in ulcerative colitis. Am J Gastroenterol 88: 852-855, 1993.

14. Turner D, Mack D, Leleiko N, et al: Severe pediatric ulcerative colitis: a prospective multicenter study of outcomes and predictors of response. Gastroenterology 138: 2282-2291, 2010.

15. Carbonnel F, Gargouri D, Lemann M, et al: Predictive factors of outcome of intensive intravenous treatment for attacks of ulcerative colitis. Aliment Pharmacol Ther 14: 273-279, 2000.

16. Kumar S, Ghoshal UC, Aggarwal R, Saraswat VA and Choudhuri G: Severe ulcerative colitis: prospective study of parameters determining outcome. J Gastroenterol Hepatol 19: 1247-1252, 2004.

17. Elloumi H, Ben Abdelaziz A, Derbel F, et al: Predictive factors of glucocorticosteroid treatment failure in severe acute idiopathic colitis. Acta Gastroenterol Belg 68: 226-229, 2005.

18. Oshitani N, Matsumoto T, Jinno Y, et al: Prediction of shortterm outcome for patients with active ulcerative colitis. Dig Dis Sci 45: 982-986, 2000

19. Cacheux W, Seksik P, Lemann M, et al: Predictive factors of response to cyclosporine in steroid-refractory ulcerative colitis. Am J Gastroenterol 103: 637-642, 2008

20. Vega R, Bertran X, Menacho M, et al: Cytomegalovirus infection in patients with inflammatory bowel disease. Am J Gastroenterol 94: 1053-1056, 1999.

21. Loftus EV Jr, Alexander GL and Carpenter HA: Cytomegalovirus as an exacerbating factor in ulcerative colitis. J Clin Gastroenterol 19: 306-309, 1994.

22. Cottone M, Pietrosi G, Martorana G, et al: Prevalence of cytomegalovirus infection in severe refractory ulcerative and Crohn's colitis. Am J Gastroenterol 96: 773-775, 2001.

23. Matsuoka K, Iwao Y, Mori T, et al: Cytomegalovirus is frequently reactivated and disappears without antiviral agents in ulcerative colitis patients. Am J Gastroenterol 102: 331-337, 2007.

24. Yoshino T, Nakase H, Ueno S, et al: Usefulness of quantitative real-time PCR assay for early detection of cytomegalovirus infection in patients with ulcerative colitis refractory to immunosuppressive therapies. Inflamm Bowel Dis 13: 1516-1521, 2007.

25. Kuwabara A, Okamoto H, Suda T, Ajioka Y and Hatakeyama K: Clinicopathologic characteristics of clinically relevant cytomegalovirus infection in inflammatory bowel disease. J Gastroenterol 42: 823-829, 2007.

26. Yoshiyama S, Miki C, Okita Y, Araki T, Uchida K and Kusunoki M: Neutrophil-related immunoinflammatory disturbance in steroid-overdosed ulcerative colitis patients. J Gastroenterol 43: 789-797, 2008. 